\title{
Local Platelet-rich plasma (PRP) injection for the treatment of delayed union after internal fixation in fractures of long bones
}

\section{Tanup Prasai, Sashmit Sharma, Kiran Prasad Rijal, Krishna Raj Khanal}

Department of Orthopaedics, Kathmandu Medical College Teaching Hospital, Sinamangal, Kathmandu, Nepal.

Correspondence: Dr. Tanup Prasai, Lecturer, Department of Orthopaedics, Kathmandu Medical College Teaching Hospital, Sinamangal, Kathmandu, Nepal.

Email: tanupprasai@gmail.com

\section{Abstract}

Introduction: Long bone fractures are among the most common orthopaedic injuries encountered. A fracture that fails to progress to union despite appropriate fixation and absence of complications presents a treatment dilemma to the surgeon. The usual solution of re-fixation with or without bone graft constitutes repeat exposure to surgery and its risks, as well as added morbidity and cost. Recent advances in molecular biology suggest Platelet rich plasma (PRP) may have bone forming potential. This study was done to determine whether PRP has any beneficial role in patients with delayed healing of long bone fractures.

Methods: A prospective interventional study was done on patients arriving at the department of orthopaedic surgery of Kathmandu Medical College with delayed union of long bone fractures after internal fixation between January 2014 and January 2017. Patients were treated with local injection of group-matched PRP directly into the fracture gap and were followed-up for six months to check for radiological signs of fracture union.

Results: A total of 10 fractures were included in 10 patients that involved four humeri, three tibiae, and three femora. Eight out of the 10 fractures united at a median time of three months after the injection. Two had non-union that required revision surgery.

Conclusion: Local Platelet rich plasma injection may constitute a 'nothing to lose, everything to gain' intermediate option before a decision for major reoperation on such patients is made.

Keywords: Delayed union; Long bone fractures; Platelet rich plasma.

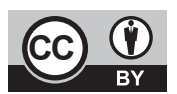

DOI: https://doi.org/10.3126/jssn.v23i1.33523

\section{Introduction}

Closed or open reduction and internal fixation with plates and/ or intramedullary nails is the standard accepted treatment for fractures of long bones. Most of these injuries can be expected to unite uneventfully in the absence of complicating factors like surgical site infection, re-trauma, or implant failure.

A fracture that is adequately fixed operatively, that does not undergo any complications, is rehabilitated properly, but still fails to demonstrate radiological union as expected, presents a dilemma for the treating surgeon. Around 10 percent of fractures treated surgically are likely to require further surgical procedures because of impaired healing. ${ }^{1,2}$ In recent times, it has been shown that the healing response can be enhanced by the use of endogenous and exogenous stimuli. $^{3}$

For fractures treated with plates and screws the option that is usually chosen is bone grafting with or without revision of the fixation. For lower limb fractures treated with intramedullary nailing, exchange nailing with a larger diameter nail is usually the preferred option. The 
above procedures, however, have their disadvantages they entail re-admission and a repeat hospital stay, a fresh surgical procedure, postoperative morbidity, and limited supply especially if bone graft is chosen. ${ }^{4}$ This translates into significant expenditure for the patient in the form of hospital expenses, operative expenses, and new implants. The patient also has to undergo the risks of anaesthesia, blood loss, and the risk of postoperative complications like infection in a previously uncomplicated procedure. Added to this may be the donor site morbidity if bone graft has to be harvested.

In recent years, there has been significant progress in the understanding and use of cellular and molecular biology of bone graft and bone graft substitutes. ${ }^{5}$ Substances such as newer compositions of hydroxyapatite, and biological molecules like recombinant human Bone Morphogenic Protein (BMP) have been advocated. BMP impregnated implants are also available and shown to have better union than non-BMP implants. ${ }^{6}$ However, widespread use of these implants is impractical because of their high cost and limited availability.

Platelet rich plasma (PRP) has been advocated in recent times as a bone graft substitute that enhances bony union. After the first description and use of PRP in the early 1990s, there is increasing interest in the application of PRP in various surgical fields ${ }^{3}$ like sports injuries, implantology, cartilage disorders, Achilles tendon injury, tennis elbow, and rotator cuff disorders. ${ }^{7-9}$

Recent studies have shown that PRP also has bone-forming properties. ${ }^{3}$ Several animal studies have shown promising results regarding bone formation in experimentally created bone or osteochondral defects treated with PRP injections alone or combined with autologous bone graft., ${ }^{4,11}$ However, there is a paucity of literature regarding the use of PRP injections to treat delayed or non-unions in humans in the clinical scenario.

We aimed to study the effect of local injection of group matched PRP in internally fixed long bone fractures which had delayed union, in terms of whether or not this accelerated the progress of fracture healing and if this method could be a viable approach to such cases.

\section{Methods}

After approval by the Institutional Review Board, an interventional study was conducted at the department of orthopaedic surgery of Kathmandu Medical College Teaching Hospital between January 2014 and January 2017. Skeletally mature patients with delayed union of long bone fractures after internal fixation with a stable implant were included in the study. Patients who had infected nonunion, non-union presenting more than nine months after surgery, fractures with unstable or broken implants as seen on radiology, and those that were lost to follow up were excluded from the study.

Delayed union was defined as the absence of radiological evidence of the progress of fracture healing on three consecutive radiographs taken two weeks apart. Union was defined as bridging of three out of four cortices of the fracture on Antero-Posterior (AP) and Lateral view radiographs.

Only radiological evaluation of union was done for the study as a clinical evaluation of the fracture site was considered unreliable with the implant still in situ. For intramedullary interlocking nails in the femur or tibia, dynamization was taken as the latest surgical procedure for calculation of interval till PRP injection.

We did not use activator in the PRP. Although many of the available studies on PRP have used it with an activator, many authors have deemed it unnecessary as they believe that the platelets get activated once they are placed in the surgical field. ${ }^{3,12}$

Patient counselling was done and informed consent was taken before the procedure. The patient was positioned on a radiolucent operating table. For the upper limb, a radiolucent arm board was used. No anaesthesia or sedation was used, and patients were not required to be kept fasting. No investigations were requested for the sake of the procedure, apart from cross-matching of the PRP. The limb was positioned under the $\mathrm{C}$-arm at the angle where the fracture gap was visible most prominently. A 16 G needle on a $50 \mathrm{cc}$ syringe loaded with PRP was introduced into the fracture gap under C-arm guidance and 20-40 cc of PRP (depending on site) was injected in and around the fracture. The needle was moved back and forth during the injection to create microtrauma at the surrounding periosteum and in the fibrous tissue inside the fracture gap. Patients were administered intramuscular diclofenac immediately after the procedure and discharged on oral analgesia.

After patients received the PRP injection, they were followed up at 2 weeks, 4 weeks, 8 weeks, 12 weeks, and 6 months. Radiographs were obtained at each visit and evaluated for union according to the aforementioned criteria.

\section{Results}

A total of 10 patients were included in the study, eight of whom were male and two were female (Figure 1). 
Sex distribution of patients

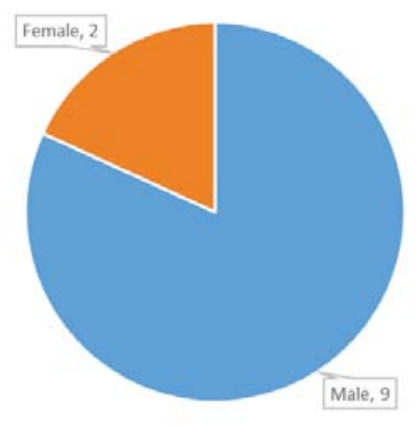

\section{Figure 1. Sex distribution of patients}

$70 \%$ of the patients were aged between 20 and 40 years and the mean age of patients was 31.4 years (Figure 2).

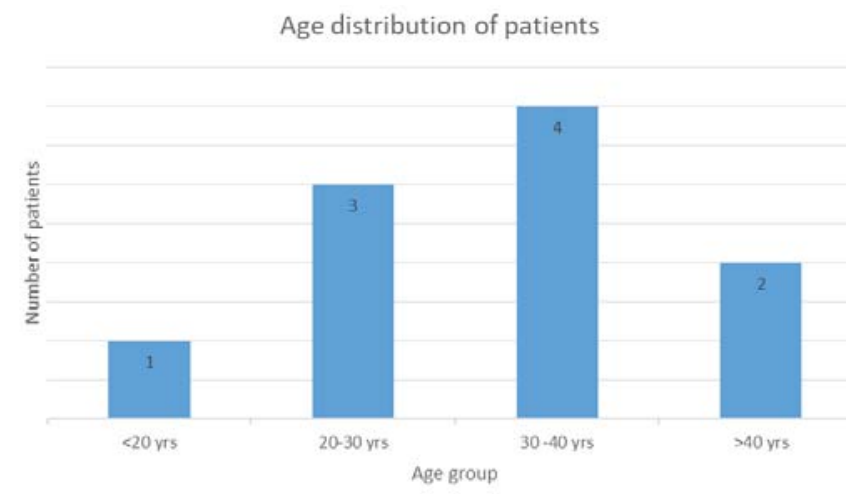

Figure 2. Age distribution of patients

Among the 10 fractures, there were four humeri, three tibiae, and three femora in the study (Figure 3).

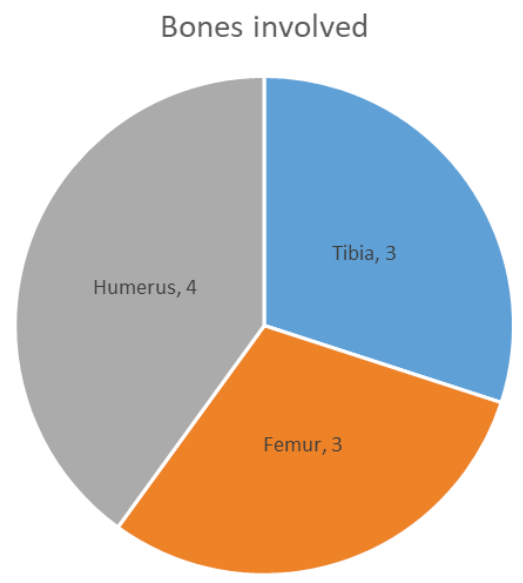

Figure 3. Bones involved
Of the total 10 bones, five had been fixed with plates and screws, three had been fixed with interlocking intramedullary nailing, one with Proximal Femoral Nail ( PFN ), and one with a proximal humerus locking plate ( Figure 4).

Implants used for original fixation

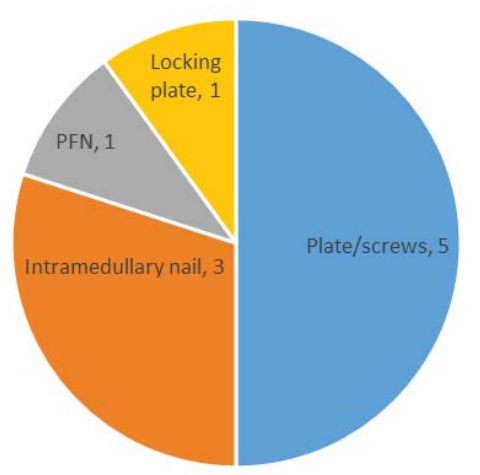

Figure 4. Implants used for fixation

Most of the patients received PRP injection between eight and twelve weeks of last surgery (Figure 5). The mean duration between the last surgical procedure and application of PRP injection was $14 \pm 5.6$ weeks.

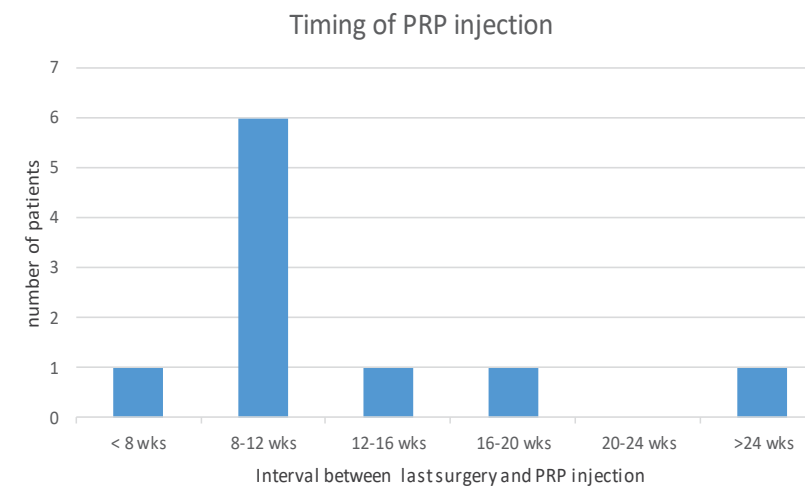

\section{Figure 5. Timing of PRP injection}

Out of the 10 patients, eight $(80 \%)$ patients got their fractures united. Two patients did not unite and had to undergo another surgical procedure with revision fixation and bone grafting. The mean time for union, in the patients who did unite, was five weeks after the injection.

There were no cases of re-fracture, compartment syndrome, or infection at the injection site.

All of the patients had painful subcutaneous swelling following the procedure, which subsided after a few hours. 


\section{Discussion}

The successful use of biological agents like rhBMP, ${ }^{6}$ and autologous bone marrow ${ }^{13}$ to enhance healing in tibial fractures in humans has been well documented. Case reports have been published regarding the use of plasma injections in delayed union ${ }^{14}$, but there is controversy in the available literature about the effectiveness of PRP in bone healing. Some studies showed a positive response with the addition of PRP in various bone regeneration procedures ${ }^{15-18}$-while some were neutral ${ }^{19-21}$ and some showed an inhibitory effect. ${ }^{22}$ In-vitro studies have shown that PRP stimulates both osteoblasts and fibroblasts, ${ }^{23}$ and that it induces logarithmic growth acceleration on stromal stem cells.

A characteristic feature of most of these studies is that autologous PRP was derived from the patient/subject's own blood. However, given the understood mechanisms by which PRP enhances union, it seems reasonable to assume that group-matched PRP concentrates would have the same effect.

Platelets contain alpha granules which undergo degranulation after activation to release growth factors. ${ }^{3}$ PRP delivers four to five times the basal concentration of platelets which releases significant quantities of growth factors, promoting healing of bone and soft tissue injuries. ${ }^{4}$ These include Platelet Derived Growth Factor (PDGF), Transforming Growth Factor Beta (TGFB), vascular endothelial growth factor, endostatin, Platelet factor 4, angiopoietins and thrombospondin 1, epidermal growth factor, and epithelial cell growth factors. In addition to growth factors, PRP contains plasma which is known to contain cell adhesion molecules like fibrin, fibronectin, and vitronectin. Fibrin can form glue and act as a scaffold for osteoconduction where stem or primary cells migrate and differentiate, synthesize type IV collagen, and potentiate healing. ${ }^{4,5}$

Graziani et al evaluated the in-vitro effect of PRP on the proliferation of osteoblast and fibroblast which are the important cell types for wound healing of soft tissues and bone. Their study found that PRP stimulates osteoblasts and fibroblasts proliferation. Similar findings were noted by various other authors on various other cells like rat calvarial bone cells, human mesenchymal progenitor cells, endothelial cells and primary periodontal ligament cells. ${ }^{24-27}$

Histomorphometric and radiological evaluation of the effect of PRP on autologous grafted mandibular bone defects has found that the volume and quality of new bone are significantly greater in PRP enriched graft sites. ${ }^{10,28}$
PRP also has been found to enhance healing of animal osteochondral and cartilaginous injuries, apart from bone healing. ${ }^{11}$

The comparative efficacy of PRP injection in delayed unions and non-unions has been studied by various authors. The effect on delayed union cases seems to be promising with various studies reporting $75-100 \%$ of delayed union cases progressing to union. ${ }^{5,14,29}$ However, the effect on nonunion cases is seen to be consistently lesser in comparison, with reported healing rates between $0 \%{ }^{26}$ to $33 \%{ }^{30}$

A comparative study of rhBMP versus PRP injections in the treatment of long bone non-union found that rhBMP may be the better option with respect to union rate $(86.7 \%$ union rate).${ }^{31}$ However, the union rate achieved by PRP $(68.3 \%)$ is reasonably good as well, and its easy availability and much cheaper cost makes it a more viable alternative than rhBMP in many scenarios.

PRP has also been shown to be effective in augmenting the ability of porous biomaterial scaffolds to repair orthotopic defects, ${ }^{32}$ such as improved spinal fusion rates when PRP was combined with Hydroxyapatite for grafting. ${ }^{33}$

In the background of such literature underlining the healing potential of biological preparations, especially based on platelet function, several authors have studied the efficacy of injections of various biological preparations to induce fracture healing. For instance, studies with percutaneous bone marrow injection in non-union and delayed union have reported rates of union from 75 to $90 \%{ }^{34-36}$

However, there is a paucity of literature about the studies done in human patients on the treatment of delayed union with isolated PRP injection. With reference to the few studies that are available for comparison, which involved the healing capacity of various platelet preparations, Bielecki et $\mathrm{al}^{5}$ evaluated 15 patients with delayed union and 20 patients with non-union treated with autologous platelet leucocyte rich gel. Union was observed in all cases of delayed union in an average of 9.3 weeks. The union rate was less and duration markedly longer for non-union cases in comparison.

In our study, $80 \%$ of the patients with delayed union got their fractures united within the study period with a median union time of 12 weeks. Of the two patients who did not unite during the study period, one was a compound fracture of the tibia which had been treated with IMIL nailing, and another was humerus fracture managed with ORIF with LCDCP. Our study showed a lower union rate than in the aforementioned study by Bielecki et al. ${ }^{5}$ 
One possible reason for the lower union rate of our study than these studies could be because of the lower concentration of platelet in our preparation, because we used the PRP available at the blood bank and did not isolate it ourselves. It has been hypothesized that the varied result of PRP treatment in non-union and delayed union may be because of the inconsistent concentration of platelets used in various studies, as the platelet concentration has been found to affect the outcome. The desired platelet count has been determined to be between 503000 to 1729000 per microlitre. Too low a concentration is found to have an inadequate healing response, while excessively high PRP concentrations may result in $\mathrm{pH}$ changes that negatively affect proliferation, ${ }^{37}$ resulting in an inhibitory effect on healing. 3,24

In our study, union was achieved in $80 \%$ of patients treated with PRP injection. However, statistical significance could not be demonstrated, presumably because of a very small sample size. PRP injection seems to be a viable option for the treatment of selected cases of long bone delayed union. It is a simple procedure which entails a minimal cost to the patient. It also avoids the risk of anaesthesia and the need for hospital admission and pre-operative investigations. The local morbidity associated with surgery and graft harvest is also avoided.

The agent being injected for treatment is just group-matched plasma, so it is not associated with any side effect or adverse reaction as with a pharmaceutical drug. Even inadvertent injection into a blood vessel or soft tissue would not cause significant damage or adverse effects. The advantage of using group-matched as opposed to autologous platelets are the cost, as the processing requires expensive equipment, and the fact that group-matched platelets can be used even in patients in whom autologous blood harvesting and use is not a good option, such as in frail or old patients, patients with low BP, seropositive patients, etc.

Our study has some inherent limitations. The sample size is small and not enough to draw any definite conclusions. The main reason for the sample size being small is because the proportion of patients with long bone fractures who have delayed union is small. In fact, it is evident that we could recruit only 10 patients over a study period of three years. Ideally, a comparative study or a randomized controlled trial with a sample size having respectable power would have allowed a more meaningful interpretation of results. This study, however, can be taken as a proof of concept study and larger multi-centric trials can be designed to recruit more patients.
Another limitation of our study is the reliance on radiological parameters alone to determine the bony union. That is not a very significant limitation because we have been relying on clinical and radiological parameters to decide fracture union in our day to day practice. Preferably, histological evaluation could have been performed to definitely evaluate the new bone formed at the injection site. However, this would require an additional surgical procedure.

One of the confounders in this study is that the definite platelet concentration in the PRP is not known, which has been suggested as an important factor influencing the outcome. Since we rely on PRP supplied by the blood bank, where quantitative analysis of platelets are routinely not done, and we do not possess platelet concentrators at our disposal, we cannot be certain about the uniformity of platelet concentration in the PRP among all the patients. We also did not use any platelet activator in our study, on the premises that the platelets become auto-activated upon placement into biological tissue.

\section{Conclusion}

We conclude that local injection of group matched PRP is a viable option in the treatment algorithm of delayed unions in fixed long bone fractures. It can be attempted as an intermediate step, before going in for formal revision fixation or grafting. We believe it represents a 'no risk, nothing to lose' option, that can potentially avoid significant expenditure, morbidity, and risk of repeat surgery.

\section{References}

1. Wilkins RM, Kelly CM: The effect of Allo- Matrix injectable putty on the outcome of long bone applications. Orthopaedics 2003;26: 567-570.

2. Simpson AHR, Mills L, Noble B: The role of growth factors and related agents in accelerating fracture healing. J Bone Joint Surg [Br] 2006; 88: 701-70

3. Lopez-Vidriero E, Goulding K A, Simon D A, Sanchez M, Johnson DH, The Use Of Platelet-Rich Plasma in Arthroscopy and Sports Medicine: Optimizing the Healing Environment. Arthroscopy, Vol 26, no.2, 2010: pp269-278

4. Broggini M, Hoffstetter W, Hunziker E, Bosshardt DD, Bornstein MM, Seto I, Weinbrich G, Buser $\mathrm{D}$, The influence of PRP on early bone formation in membrane protected defects. A histological and histomorphometric study in the rabbit calvaria. Clin Implant Dent Relat Res.2011 Mar; 13(1):1-12 
5. Bielecki T, Gazdzik TS, Szczepanski T. Benefit of percutaneous injection of autologous plateletleukocyte rich gel in patients with delayed union and non union. Eur Surg Res 2008;40:289-96

6. Govender $\mathrm{S}$ et al, Recombinant human bone morphogenic protein-2 for treatment of open tibial fractures: a prospective, controlled, randomized study of four hundred and fifty patients. J Bone Joint Surg Am. 2002 Dec; 84-A(12): 2123-34

7. Sanchez M, Anitua E, Azofra J, Andia I, Padilla S, Mujika I. Comparison of surgically repaired Achilles tendon tears using platelet-rich fibrin matrices. Am J Sports Med 2007;35:245-251

8. Arnoczky SP, Anderson L, Fanelli G, Ho S, Mishra A, Sgaglione N. The role of platelet-rich plasma in connective tissue repair. Orthopedics Today 2009;26:29

9. Gosen T, Sluimer J. Prospective randomized study on the effect of autologous platelets injection in lateral epicondylitis compared with corticosteroid injection. Poster P25-444. Presented at: 13th Congress of the European Society of Sports Traumatology, Knee Surgery and Arthroscopy (ESSKA), Porto, Portugal, May 21-24, 2008.

10. Gerard D, Carlson ER, Gotcher JF,Jacobs M. Effects of platelet-rich plasma on the healing of autologous bone grafted mandibular defects in dogs. J Oral Maxillofac Surg.2006 Mar;64(3):443-51

11. Y Sun, Y Feng, CQ Zhang, SB Chen, XG Cheng. The regenerative effect of platelet- rich plasma on healing in large osteochondral defects. International Orthopaedics (SICOT) (2010) 34: 589-597

12. Lowery GL, Kulkarni S, Pennisi AE: Use of autologous growth factors in lumbar spinal fusion. Bone 1999; 2: 47-502-21

13. Braly HL, O'Connor DP, Brinker MR. Percutaneous autologuos bone marrow injection in the treatment of distal meta-diaphyseal tibial ninunions and delayed unions. J Orthop Trauma.2013 Sep;27(9):527-33

14. Seijas R, Santana-Suarez RY, Garcia-Balletbo M, Cusco X, Ares O, Cugat R. Delayed union of clavicle treated with plasma rich in growth factors. Acta Orthop. Belg,2010,76,689-693

15. Kassolis JD, Reynolds MA. Evaluation of the adjunctive benefits of platelet-rich plasma in subantral sinus augmentation. J Craniofac Surg 2005; 16:280-287.
16. Aghaloo TL, Moy PK, Freymiller EG. Evaluation of plateletrich plasma in combination with anorganic bovine bone in the rabbit cranium: a pilot study. Int J Oral Maxillofac Implants 2004; 19:59-65

17. Ouyang XY, Qiao J. Effect of platelet-rich plasma in the treatment of periodontal intrabony defects in humans. Chin Med J 2006; 119:1511-1521

18. Suba Z, Takacs D, Gyulai-Gaal S, Kovacs K. Facilitation of beta-tricalcium phosphate-induced alveolar bone regeneration by platelet-rich plasma in beagle dogs: a histologic and histomorphometric study. Int J Oral Maxillofac Implants 2004; 19:832838

19. Aghaloo TL, Moy PK, Freymiller EG. Investigation of platelet-rich plasma in rabbit cranial defects: a pilot study. J Oral Maxillofac Surg 2002; 60:1176-1181

20. Furst G, Gruber R, Tangl S, et al. Sinus grafting with autogenous platelet-rich plasma and bovine hydroxyapatite. A istomorphometric study in minipigs. Clin Oral Implants Res 2003; 14:500-508

21. Grageda E, Lozada JL, Boyne PJ, Caplanis N, McMillan PJ. Bone formation in the maxillary sinus by using platelet-rich plasma: an experimental study in sheep. J Oral Implantol 2005; 31:2-17

22. Choi BH, Im CJ, Huh JY, Suh JJ, Lee SH. Effect of plateletrich plasma on bone regeneration in autogenous bone graft. Int J Oral Maxillofac Surg 2004; 33:56-59

23. Graziani F, Ivanovski S, Cei S, Ducci F, Tonetti M, Gabriele M. The in vitro effect of different PRP concentrations on osteoblasts and fibroblasts. Clin. Oral. Impl. Res. 17,2006;212-219

24. Lucarelli F, Beccheroni A,Donati D, Sangiorgi L, Cenacchi A, Del Vento AM, Meotti C, Bertoja AZ, Giardino R, Fornasari PM, Mercuri M, Picci P. Biomaterials. 2003 Aug;24(18)3095-100.

25. Soffer, E., Ouhayoun, J.P., Dosquet, C., Meunier, A. \& Anagnostou, F. (2004) Effects of platelet lysates on select bone cell functions. Clinical Oral Implants Research 15: 581-588

26. Kilian, O., Flesch, I., Wenisch, S., Taborski, B., Jork, A., Schnettler, R. \& Jonuleit, T. (2004) Effects of platelet growth factors on human mesenchymal stem cells and human endothelial cells in vitro. European Journal of Medical Research 9:337-344 
27. Okuda, K., Kawase, T., Momose, M., Murata, M., Saito, Y., Suzuki, H., Wolff, L.F. \& Yoshie, H. (2003) Platelet-rich plasma contains high levels of plateletderived growth factor and transforming growth factorbeta and modulates the proliferation of periodontally related cells in vitro. Journal of Periodontology 74:849-857

28. Marx, R.E., Carlson, E.R., Eichstaedt, R.M., Schimmele, S.R., Strauss, J.E. \& Georgeff, K.R. (1998) Platelet-rich plasma: growth factor enhancement for bone grafts. Oral Surgery, Oral Medicine, Oral Pathology, Oral Radiology, and Endodontics 85:638-646.

29. Say F, Turkeli E, Bulbul M. Is platelet rich plasma injection an effective choice in cases of non union? Acta Chirurgiae Orthopaedicae et Traumatologiae Cechosl, 81, 2014,p 340-345

30. Guo Y, Qiu J, Zhang C. Follow up study on platelet rich plasma in repairing chornic wound non union of lower limbs in 47 cases. Zhongguo Xiu Fu Chong Jian Wai Ke Za Zhi. 2008 Nov;22(11):1301-5.

31. Calori GM, Tagliabue L, Gala L, D’Imporzano M, Peretti G, Albisetti W. Application of rhBMP-7 and platelet-rich plasma in the treatment of long bone non unions: A prospective randomized clinical study on 120 patients. Injury, Int. J Care Injured (2008) 39,1391-1402

32. Rai B, Oest ME, Dupont KM, Ho KH et al. combination of platelet rich plasma with polycaprolactomnetricalcium phosphate scaffolds for segmental bone defect repair. J Biomed Mater Res, 2007 ( 81A): 888-899

33. Kamoda H, Yamashita M, Ishikawa T, Miyagi M et al. Platelet rich plasma combined with hydroxyapatite for lumbar interbody fusion promoted bone formation and decreased an inflammatory pain neuropeptide in rats. Spine 2012;37(20):1727-33

34. Hernigou P, Poignard A, Beaujean F, et al. Percutaneous autologous bone-marrow grafting for nonunions. Influence of the number and concentration of progenitor cells. J Bone Joint Surg Am. 2005;87:1430-1437
35. Goel A, Sangwan SS, Siwach RC, et al. Percutaneous bone marrow grafting for the treatment of tibial nonunion. Injury. 2005;36:203-206 8-10

36. Bhargava R, Sankhla S, Gupta A, et al. Percutaneous autologus bone marrow injection in the treatment of delayed or nonunion. Indian J Orthop. 2007;41:67-71

37. Liu, Y., Kalen, A., Risto, O. \& Wahlstrom, O. (2002) Fibroblast proliferation due to exposure to a platelet concentrate in vitro is $\mathrm{pH}$ dependent. Wound Repair and Regeneration 10: 336-340 